Quim. Nova, Vol. 34, No. 6, 996-1000, 2011

\title{
FORMAÇÃO DE CARBAMATO DE ETILA EM AGUARDENTES RECÉM-DESTILADAS; PROPOSTA PARA SEU CONTROLE
}

\author{
Carlos Alexandre Galinaro e Douglas Wagner Franco* \\ Instituto de Química de São Carlos, Universidade de São Paulo, CP 780, 13560-970 São Carlos - SP, Brasil
}

Recebido em 1/10/10; aceito em 17/1/11; publicado na web em 29/3/11

\begin{abstract}
ETHYL CARBAMATE FORMATION IN RECENTLY DISTILLED SUGAR CANE SPIRITS; PROPOSAL FOR ITS CONTROL. The commercial sugar cane spits redistillation decreased up to $92,5 \%$ their ethyl carbamate (EC) original content. Quantitative analysis of EC in 15 samples of sugar cane spirit (alembic and column), fresh distilled and collected in situ demonstrated that the urethane is formed mostly after distillation. The average time to achieve the complete EC formation is independent of the diffuse light presence and of the distillation apparatus used. The $k_{o b s}$ for urethane formation at $25^{\circ} \mathrm{C}$ was calculate as $(3,3 \pm 0,5) \times 10^{-5} / s$ and the activation parameters are: $\Delta \mathrm{H}^{\ddagger} 34 \mathrm{kcal} / \mathrm{mol} ; \Delta \mathrm{S}^{\ddagger}-69 \mathrm{cal} / \mathrm{mol} \mathrm{K}$; and $\Delta \mathrm{G}^{\ddagger} 54 \mathrm{kcal} / \mathrm{mol}$.
\end{abstract}

Keywords: ethyl carbamate; formation; sugar cane spirit.

\section{INTRODUÇÃO}

$\mathrm{O}$ carbamato de etila ou uretana $\left(\mathrm{CE}, \mathrm{C}_{2} \mathrm{H}_{5} \mathrm{COONH}_{2}\right)$, naturalmente presente em queijos, iogurtes, molhos de soja, pães, e bebidas alcoólicas (vinhos, saque, uísque, aguardente, vodca, rum, cerveja, cidras, tiquira), é uma substância com reconhecido potencial carcinogênico. ${ }^{1-9}$ Foi utilizado como hipnótico, anestésico e como cossolvente em algumas sínteses de fármacos. Em testes de toxicidade em animais causou edemas pulmonares, papilomas de pele, linfomas malignos, hepatomas, carcinomas mamários, tumores de ovário, hemangiomas hepáticos, tumores melanóticos de pele e de íris, entre tantos outros. ${ }^{1-9}$

Hoje, Canadá, EUA, França, República Tcheca, Alemanha e Suíça controlam a presença de CE em bebidas alcoólicas. ${ }^{6}$

No Brasil, a presença de carbamato de etila em aguardentes é monitorada pela Instrução Normativa $n^{\circ} 13$, de 29/6/2005. ${ }^{10}$ Este documento estabelecia que o limite de $150 \mu \mathrm{g} / \mathrm{L}$ deveria ser implantado em junho de 2010. ${ }^{10}$ Recentemente este prazo foi dilatado para 30/6/2012. ${ }^{11}$ É desconhecido o controle de CE em alimentos no país.

As vias de formação e os precursores de uretana em alimentos e bebidas dependem do tipo e do seu respectivo processamento. ${ }^{3-7,12}$

Em vinhos a presença de carbamato de etila está relacionada com o tipo de levedura e com os subprodutos do seu metabolismo. $.^{3-6} \mathrm{Ex}-$ perimentos com soluções modelo de vinho fortificadas demonstraram que a ureia (utilizada como nutriente durante a fermentação ou oriunda de leveduras), a citrulina e os N-carbamil fosfatos são os principais precursores de CE. Estes por reação com o etanol poderão levar à formação de CE..$^{1,13-20}$ Além disto, a temperatura da fermentação, o $\mathrm{pH}$, a luz e o tempo de estocagem estão relacionados com aumento nos teores de CE. ${ }^{2-9,20-22}$

Para bebidas destiladas como uísque, rum e destilados de frutas com caroço (stone-fruit brandies) apenas uma pequena concentração de uretana presente no mosto fermentado é incorporada ao destilado final, ${ }^{1,3-6}$ sendo o íon cianeto relatado como o principal precursor de CE. ${ }^{1,3-6,12,21-24} \mathrm{O}$ cianeto $\left(\mathrm{CN}^{-}\right)$, ânion do acido cianídrico, seria formado quando glicosídeos cianogênicos (Figura 1) presentes na cevada (epiheterodendrin), mandioca, aveia e em cereais (linamarin

*e-mail: douglas@iqsc.usp.br e lotaustralin), no centeio e no trigo (dhurrin), na maçã (amygdalin), manga e no arroz, por exemplo, ${ }^{25}$ são degradados enzimaticamente liberando cianeto, passível de ser oxidado a cianato $\left(\mathrm{CNO}^{-}\right)$o qual reage com o etanol para formar o CE..$^{5,6,12,24}$ Embora, até o presente momento não esteja descrito na literatura qual o glicosídeo cianogênico presente na cana-de-açúcar, é bem razoável admitir a sua existência neste vegetal. ${ }^{25}$<smiles>N#C[C@@H](O[C@@H]1O[C@H](CO)[C@@H](O)[C@H](O)[C@H]1O)c1ccc(O)cc1</smiles>

Dhurrin<smiles>CC(C)(C#N)O[C@@H]1O[C@H](CO)[C@@H](O)[C@H](O)[C@H]1O</smiles>

Linamarin
Figura 1. Exemplos de alguns glicosídeos cianogênicos

Em função do seu elevado ponto de ebulição $\left(\mathrm{PE} \approx 182-184{ }^{\circ} \mathrm{C}\right)^{9,26}$ o CE não deveria ser transferido ao destilado em uma destilação bem conduzida. É conhecido que amostras de uísque apresentaram redução na concentração de uretana ao serem redestiladas..$^{17,23}$

No presente trabalho são descritos e discutidos os efeitos de uma redestilação simples na redução da concentração de carbamato de etila em aguardentes. Também são descritos o acompanhamento da formação de uretana em aguardentes recém-destiladas em função do tempo de armazenagem, bem como a influência da luz, do sistema de destilação (alambique e coluna) e da temperatura neste processo.

\section{PARTE EXPERIMENTAL}

\section{Amostras de aguardente coletadas}

Foram analisadas 15 amostras de aguardente coletadas in loco em diferentes destilarias, sendo 6 amostras destiladas em alambique de cobre e 9 em coluna de aço inox. Das 15 amostras de aguardente, em 7 amostras ( 3 destiladas em alambique e 4 em coluna) não foi detec- 
tada a presença de carbamato de etila em concentrações superiores ao limite de quantificação (LQ = $30 \mu \mathrm{g} / \mathrm{L})$ da metodologia utilizada. ${ }^{26,27}$ Um questionário contendo as informações sobre a origem da canade-açúcar, o tipo de colheita, o sistema de destilação, a condução da fermentação (adição ou não de nutrientes) foi respondido pelos produtores durante a coleta destas amostras.

No momento das coletas, as aguardentes foram colocadas em garrafas de vidro, envoltas por papel alumínio, armazenadas em banho de gelo $\left(\approx 2,0 \pm 0,5^{\circ} \mathrm{C}\right)$ e transportadas em recipiente térmico para o laboratório, onde foram mantidas a baixa temperatura e prontamente utilizadas. Algumas amostras foram analisadas quanto ao teor de $\mathrm{CE}$ na presença e na ausência de luz.

Foram feitas análises a temperatura ambiente $\left(27 \pm 2^{\circ} \mathrm{C}\right)$ e em temperaturas de $15 \pm 0,2{ }^{\circ} \mathrm{C}, 25 \pm 0,2^{\circ} \mathrm{C}$ e $35 \pm 0,2^{\circ} \mathrm{C}$. A temperatura ambiente refere-se à temperatura de um ambiente termostatizado utilizando-se ar condicionado. $\mathrm{O}$ controle da temperatura a $15^{\circ} \mathrm{C}$ foi efetuado utilizando-se um banho termostatizado da marca Tecnal, modelo TE 184. Para as temperaturas 25 e $35^{\circ} \mathrm{C}$ utilizou-se um banho seco (dry bath) da marca LGC Biotecnologia, modelo DB-100.

\section{Reagentes}

Os reagentes utilizados foram de grau analítico (Sigma-Aldrich, Mallinckrodt). O carbamato de etila e o carbamato de propila (padrão interno) foram adquiridos da Sigma-Aldrich. Estes foram diluídos em solução de acetona (Mallinckrodt) e água deionizada (Milli-Q) na proporção de 1:1, para a preparação da solução estoque 4,30 mg/L.

\section{Metodologia}

As análises de carbamato de etila foram efetuadas seguindo metodologia descrita na literatura. ${ }^{26,27}$ Utilizou-se um cromatógrafo para fase gasosa (GC/MS) Shimadzu, modelo17-A, com detector de massas Shimadzu QP-5050, usando impacto eletrônico de 70 eV como modo de ionização, operando no modo SIM (monitoramento de íon seletivo com razão $\mathrm{m} / \mathrm{z}, 62$ ). As amostras foram quantificadas por meio de uma curva analítica $(30-1000 \mu \mathrm{g} / \mathrm{L})$ com coeficiente de correlação de 0,996 . Os limites de detecção (LD) e quantificação (LQ) para o CE foram de 10,0 e $30 \mu \mathrm{g} / \mathrm{L}$, respectivamente, calculados com base na razão sinal/ruído. ${ }^{28}$

\section{Redestilação das aguardentes comerciais}

A redestilação foi efetuada utilizando-se um balão de 2,0 L e uma coluna tipo Vigreux, de $31 \mathrm{~cm}$ de comprimento, $1,5 \mathrm{~cm}$ de d.i. e com 7 pratos teóricos, calculados segundo o método de McCAbe-Thiele. ${ }^{29}$ A média da taxa de destilação foi de $1,7 \pm 0,3 \mathrm{~mL} / \mathrm{min}$.

As aguardentes originais (aguardente comercial) e as amostras obtidas da redestilação das aguardentes comerciais foram analisadas quanto ao teor de carbamato de etila. Segundo a legislação, ${ }^{10}$ a concentração de $\mathrm{CE}$ em aguardentes é expressa em microgramas por litro $(\mu \mathrm{g} / \mathrm{L})$ de aguardente, não importando o teor alcoólico (\% v/v) da amostra. Entretanto, para facilitar a comparação dos teores de CE antes e após a destilação, optou-se por expressar os resultados (Tabela 1) em $\mu \mathrm{g} / 100 \mathrm{~mL}$ A.A..

Nos testes de redestilação foram utilizadas 14 amostras de aguardentes comerciais, adquiridas no mercado, portanto com teor de $\mathrm{CE}$ estabilizado. Também com o objetivo de verificar a influência do teor alcoólico (\% v/v), nos teores de carbamato de etila que acompanham o destilado, foram obtidas por diluição de uma aguardente (Tabela 1 , amostra O com teor alcoólico de $47 \% \mathrm{v} / \mathrm{v}$ ) amostras com diferentes teores alcoólicos: 8,$0 ; 24 ; 28 ; 33 ; 38$ e $42 \% \mathrm{v} / \mathrm{v}$, que posteriormente foram analisadas quanto ao teor de uretana.

\section{Irradiação em bancada fotoquímica}

Duas das amostras de aguardente comerciais, escolhidas aleatoriamente, foram submetidas à irradiação em bancada fotoquímica em diferentes comprimentos de onda $(250,280,310,330,410,440$ e $500 \mathrm{~nm}$ ) durante $3 \mathrm{~h}$. Posteriormente, foram analisadas quanto ao teor de CE, na tentativa de se observar alguma variação no teor final deste contaminante. O teor determinado foi comparado com amostra idêntica (não irradiada) ao abrigo da luz.

As irradiações foram realizadas em uma bancada fotoquímica da marca Oriel, modelo 68805 Universal, com lâmpada de xenônio de $200 \mathrm{~W}$, com filtros de interferências apropriados Oriel. O cálculo da intensidade de radiação incidente pelo uso do actinômetro químico $\left(\mathrm{K}_{3}\left[\mathrm{Fe}\left(\mathrm{C}_{2} \mathrm{O}_{4}\right)_{3}\right]\right.$, tris(oxalato) ferrato(III) de potássio, $0,15 \mathrm{~mol} / \mathrm{L}$ ) foi realizado segundo os parâmetros da literatura, ${ }^{30}$ e foi de $1,30 \mathrm{x}$ $10^{-7}$ Einstein/min.

\section{Experimentos cinéticos}

Os valores de $t_{1 / 2}$, tempo de meia-vida do processo, foram calculados a partir de gráficos da concentração de carbamato de etila ([CE]) em função do tempo de reação. ${ }^{31}$ Considerou-se $t_{1 / 2}$ como o tempo correspondente à formação de metade da concentração máxima de uretana $\left([\mathrm{CE}]_{\infty}\right)$. $^{31}$

Embora se desconhecendo a natureza do(s) precursor(es) do carbamato de etila $(P C E)$ nesta matriz, sua concentração é, muito inferior à do etanol $([\mathrm{EtOH}])$ presente nas aguardentes, $[\mathrm{PCE}]<<<[E t O H]$. Outra possibilidade que deve ser considerada é o fato deste precursor $P C E$ se converter em uma forma reativa " $P C E$ " (Esquema 1).

\section{$P C E \stackrel{\mathrm{k}^{\prime}}{\longrightarrow}$ "PCE" \\ Esquema 1}

Assim, considerando o conhecimento acumulado sobre a reação de formação de carbamato de etila em outras bebidas, ${ }^{15,18,32,33}$ esta reação seria de primeira ordem em relação às concentrações do precursor e do etanol (Esquema 2).

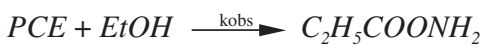

Esquema 2

Entretanto, nas aguardentes aqui estudadas a concentração molar de etanol oscilou entre 7,0 a 9,3 mol/L. Nestas condições é interessante considerar a possibilidade da reação ser independente da concentração de etanol e dos precursores, mas dependente da concentração do precursor em sua forma reativada " $P C E$ ', ou seja, dependente da constante $k$ '.

As constantes $k_{o b s}$ foram calculadas por meio de gráficos ln $\left([\mathrm{CE}]_{\infty}-[\mathrm{CE}]_{\mathrm{t}}\right)$ versus tempo, os quais se demonstraram lineares por pelo menos 2 meia-vidas. Para o cálculo dos parâmetros cinéticos as temperaturas foram controladas em $0,1{ }^{\circ} \mathrm{C}$, utilizando-se uma graduação alcoólica constante.

Os parâmetros de ativação $\left(\Delta H^{\ddagger}, \Delta \mathrm{S}^{\ddagger}\right.$ e $\left.\Delta \mathrm{G}^{*}\right)$ foram calculados resolvendo-se graficamente as seguintes equações: ${ }^{31}$

$$
\begin{gathered}
\ln k=\ln \frac{k_{B}}{k_{h}}+\ln T-\frac{\Delta H^{\ddagger}}{R T}+\frac{\Delta S^{\ddagger}}{R} \\
\mathrm{e} \\
\Delta G^{\ddagger}=\Delta H^{\ddagger}+T \Delta S^{\ddagger}
\end{gathered}
$$

onde, $k_{o b s}$ é a constante de velocidade observada em determinada 
temperatura, $k_{B}$ a constante de Boltzmann $\left(1,38065 \times 10^{-23} \mathrm{~J} / K\right), k_{h}$ a constante de Planck $\left(6,626 \times 10^{-34} J s\right)$, T é a temperatura (Kelvin), $\Delta H^{\ddagger}$ variação de entalpia, $\Delta S^{\ddagger}$ é a variação de entropia, $\Delta G^{\ddagger}$ é a energia livre de Gibbs, $R$ é a constante universal dos gases $(1,987 \mathrm{cal} / \mathrm{K} \mathrm{mol}){ }^{31}$

\section{RESULTADOS E DISCUSSÃO}

\section{Redestilação de amostras comerciais}

A Tabela 1 apresenta o teor alcoólico e as concentrações de uretana, expressas em $\mu \mathrm{g} / 100 \mathrm{~mL}$ A.A., nas amostras comerciais de aguardentes antes e após serem redestiladas, bem como a porcentagem da redução do teor de CE após a redestilação. No processo de dupla destilação (bidestilação ou redestilação) realizado em alambique, a destilação é realizada sem cortes das frações alcoólicas, até que se obtenha um destilado com teor alcoólico entre 25 a $27 \%$ de álcool em volume (low wines). Este destilado ("flegma") é então submetido a uma nova destilação, obtendo-se as frações cabeça, coração (aguardente) e cauda. ${ }^{34-36}$

Neste estudo as amostras de aguardentes comerciais (fração coração) foram novamente destiladas, no intuito de se avaliar a eficiência desta segunda destilação na redução de teor de uretana. Este experimento se assemelha ao efetuado pela indústria quando utiliza cachaça industrial como "matéria-prima" para a produção de aguardente bidestilada.

As amostras de aguardente ao serem redestiladas apresentaram uma redução de 66,0 a 92,5\% do teor de CE (original). A variação no teor alcoólico de uma aguardente (aguardente $\mathrm{O}$, Tabela 1) não influiu significativamente no teor final do $\mathrm{CE}$, quando comparado com a amostra comercial (aguardente $\mathrm{O}$, Tabela 1) redestilada sem a prévia diluição. Assim, variando-se o teor alcoólico de 8,0 a 47\% v/v, a redução no teor de CE nas amostras de aguardente foi de 80,3 a $87,7 \%$, com uma porcentagem média de $83,9 \%$. Este resultado é semelhante ao verificado para amostras de uísque $(84 \%),{ }^{24}$ mas superior ao observado para amostras de destilados que foram filtradas em carvão vegetal, onde se informa ter ocorrido uma redução máxima de $47 \%$ da concentração de uretana..$^{37}$

Estas observações, embora restritas à fração coração, dão suporte à proposta anterior ${ }^{34}$ envolvendo a dupla destilação de aguardentes, objetivando a redução dos teores de cobre e acidez total, para a redução no teor de CE em aguardentes. É interessante salientar que, segundo a literatura, ${ }^{35,36}$ a redestilação não afeta significativamente as propriedades sensoriais da aguardente.

\section{Experimentos com aguardentes recém-destiladas}

Os experimentos foram conduzidos com amostras de aguardentes recém-destiladas, coletadas em diferentes produtores.

A Figura 2 apresenta a curva de formação de CE em função do tempo, para uma mesma aguardente a temperatura ambiente $\left(27 \pm 2{ }^{\circ} \mathrm{C}\right)$, na presença e na ausência de luz difusa (luz solar). Como se observa as duas curvas apresentam perfil semelhante e são idênticas, dentro dos limites de erro experimental, com respeito aos teores finais de uretana. A reprodução deste resultado utilizando-se aguardentes de outros produtores sugere que, contrariamente ao descrito na literatura para outros destilados ${ }^{4-9,21,22,38,39}$ e no caso das aguardentes aqui estudadas, é pouco provável a ocorrência de um precursor ativado fotoquimicamente.

Também foram concordantes dentro do erro experimental (RDS), os teores de $\mathrm{CE}$ em amostras de aguardentes comerciais submetidas à irradiação em bancada fotoquímica com luz de diferentes comprimentos de onda (250 a $500 \mathrm{~nm}$ ) (Tabela 2), demonstrando que caso exibisse um precursor fotoquímico nestas amostras, o mesmo não foi ativado.
Tabela 1. Amostras de aguardente (A, B, ..., O6), teor alcoólico (\% v/v), concentração de carbamato de etila ([CE]) nas amostras de aguardente antes (original) e após serem redestiladas em $\mu \mathrm{g} / 100 \mathrm{~mL} \mathrm{A.A.}{ }^{\mathrm{a}}$, e a porcentagem (\%) de redução de CE após a redestilação

\begin{tabular}{|c|c|c|c|c|}
\hline Aguardente & $\begin{array}{c}\text { Teor } \\
\text { alcoólico } \\
(\% \mathrm{v} / \mathrm{v})\end{array}$ & $\begin{array}{c}{[\mathrm{CE}]} \\
{\text { (original }{ }^{\mathrm{b}}} \\
(\mu \mathrm{g} / 100 \mathrm{~mL} \\
\text { A.A.) }\end{array}$ & $\begin{array}{c}{[\mathrm{CE}]} \\
\text { (redestilada) } \\
(\mu \mathrm{g} / 100 \mathrm{~mL} \\
\text { A.A. })\end{array}$ & $\begin{array}{l}\text { Redução de } \\
\text { CE (\%) após } \\
\text { a redestilação }\end{array}$ \\
\hline A & 38 & 32,1 & 10,7 & 66,7 \\
\hline B & 40 & 71,7 & 24,4 & 66,0 \\
\hline $\mathrm{C}$ & 41 & 66,2 & 20,6 & 68,9 \\
\hline D & 44 & 25,6 & 6,60 & 74,3 \\
\hline $\mathrm{E}$ & 39 & 77,6 & 13,9 & 82,0 \\
\hline $\mathrm{F}$ & 38 & 124 & 37,5 & 69,7 \\
\hline G & 41 & 215 & 30,9 & 85,6 \\
\hline $\mathrm{H}$ & 59 & 127 & 11,7 & 90,8 \\
\hline $\mathrm{J}$ & 40 & 101 & 17,0 & 83,1 \\
\hline K & 44 & 97,5 & 17,6 & 81,9 \\
\hline $\mathrm{L}$ & 41 & 29,7 & 7,80 & 73,6 \\
\hline M & 38 & 30,7 & 6,90 & 77,5 \\
\hline $\mathrm{N}$ & 48 & 117 & 8,70 & 92,5 \\
\hline $\mathrm{O}^{\mathrm{c}}$ & 47 & 120 & 4,70 & 82,3 \\
\hline $\mathrm{O} 1$ & 42 & 120 & 17,3 & 85,6 \\
\hline $\mathrm{O} 2$ & 38 & 120 & 22,9 & 80,9 \\
\hline $\mathrm{O} 3$ & 33 & 120 & 17,0 & 85,8 \\
\hline $\mathrm{O} 4$ & 28 & 120 & 18,4 & 84,7 \\
\hline O5 & 24 & 120 & 14,7 & 87,7 \\
\hline O6 & 8,0 & 120 & 23,6 & 80,3 \\
\hline
\end{tabular}

${ }^{a}$ Concentração em $\mu \mathrm{g} / 100 \mathrm{~mL}$ A.A. $=(10 \div$ teor alcoólico em \%v/v $)$ x concentração de carbamato de etila em $\mu \mathrm{g} / \mathrm{L}$.; ${ }^{\text {c}}$ carbamato de etila na aguardente comercial. 'Aguardente diluída, onde foi reduzido o teor alcoólico, obtendo-se as aguardentes $\mathrm{O} 1, \mathrm{O} 2, \mathrm{O} 3, \mathrm{O} 4, \mathrm{O} 5$ e $\mathrm{O} 6$.

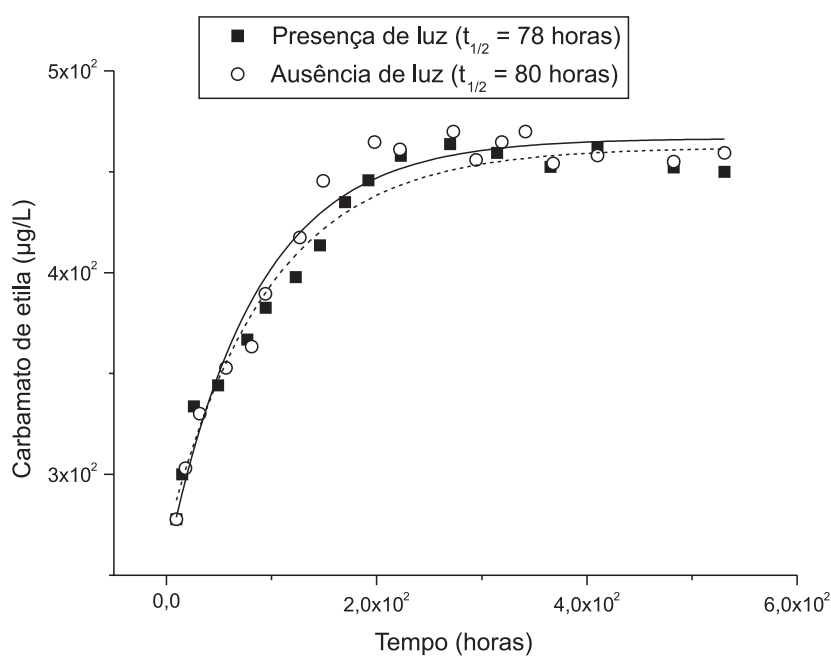

Figura 2. Carbamato de etila $(\mu \mathrm{g} / \mathrm{L})$ em função do tempo ( $h$ ) em uma amostra de aguardente (oriunda de um mesmo produtor) analisada na presença (ם) e na ausência de luz difusa $(\bigcirc)$ a temperatura ambiente $\left(27 \pm 2{ }^{\circ} \mathrm{C}\right)$, coletada in loco

Os resultados obtidos para amostras de aguardente coletadas na saída do destilador, oriundas do mesmo produtor ou não, indicam que mais de $60 \%$ do teor de carbamato de etila se forma após destilação, que a concentração máxima é atingida no prazo médio de 7 dias após a obtenção do destilado e, a partir desta data, este valor ([CE $\left.]_{\infty}\right)$ permanece inalterado. 
Tabela 2. Concentração de carbamato de etila ([CE]) em $\mu \mathrm{g} / \mathrm{L}$ em aguardentes comerciais após irradiação em bancada fotoquímica em diferentes comprimentos de onda, durante $3 \mathrm{~h}$

\begin{tabular}{ccc}
\hline $\begin{array}{c}\text { Comprimento } \\
\text { de onda }(\mathrm{nm})\end{array}$ & $\begin{array}{c}\text { [CE] Aguardente } \\
\text { não envelhecida }^{\mathrm{a}}\end{array}$ & $\begin{array}{c}\text { [CE] Aguardente } \\
\text { envelhecida }^{\mathrm{b}}\end{array}$ \\
\hline Não irradiada & 289 & 244 \\
250 & 253 & 260 \\
280 & 275 & 251 \\
310 & 255 & 250 \\
330 & 241 & 250 \\
410 & 279 & 253 \\
440 & 281 & 251 \\
500 & 292 & 257 \\
Média [CE] & 271 & 252 \\
\hline
\end{tabular}

Desvio padrão relativo (RDS, residual standard desviation): $\mathrm{a}=7,0 \%$; $\mathrm{b}=2,0 \%$. $^{28}$

A Figura 3 ilustra a evolução do teor de carbamato de etila em amostras coletadas in loco, provenientes do mesmo mosto fermentado, destiladas em alambique de cobre e em coluna de aço inox, em função do tempo. Nota-se que independente da destilação ser processada em alambique ou em coluna, atinge-se um máximo de $[\mathrm{CE}]_{\infty}$ após um período médio de aproximadamente 7 dias, com tempos de meia-vida de respectivamente 85 e $80 \mathrm{~h}$.

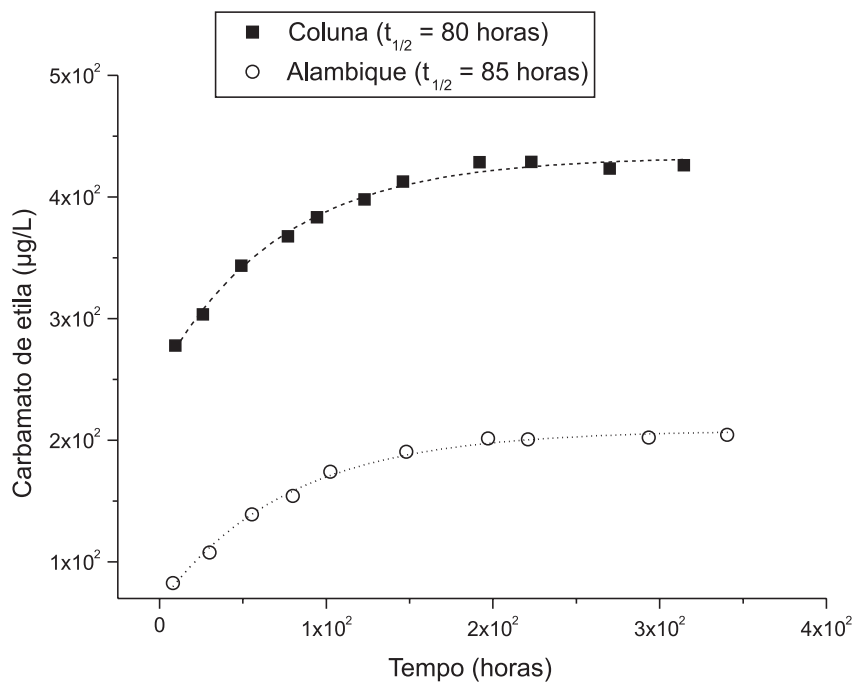

Figura 3. Carbamato de etila $(\mu \mathrm{g} / \mathrm{L})$ em função do tempo $(h)$ em aguardentes oriundas de um mesmo produtor e mesmo mosto fermentado, destiladas em alambique de cobre $(\bigcirc)$ e em coluna de aço inox $(\mathbf{\square})$, a temperatura ambiente $\left(27 \pm 2{ }^{\circ} \mathrm{C}\right)$, coletadas in loco

O mesmo vinho (mosto fermentado de cana-de-açúcar), destilado em alambique e em coluna (Figura 3), conduziu a destilados com teores de carbamato de etila finais diferentes, respectivamente, 204 e $423 \mu \mathrm{g} / \mathrm{L}$. Isto está em concordância com relatos anteriores ${ }^{7,8,26}$ que indicam menor teor de uretana para aguardentes destiladas em alambique em relação às destiladas em coluna.

As curvas de [CE] versus tempo, obtidas para destilados de diferentes produtores (Figura 4), são bastante semelhantes, conduzindo a valores de meia-vida para o processo da mesma ordem de grandeza.

A evolução do teor de CE nestas amostras também foi acompanhada a diferentes temperaturas. A Figura 5 claramente evidencia a influência da temperatura na evolução da formação de uretana.

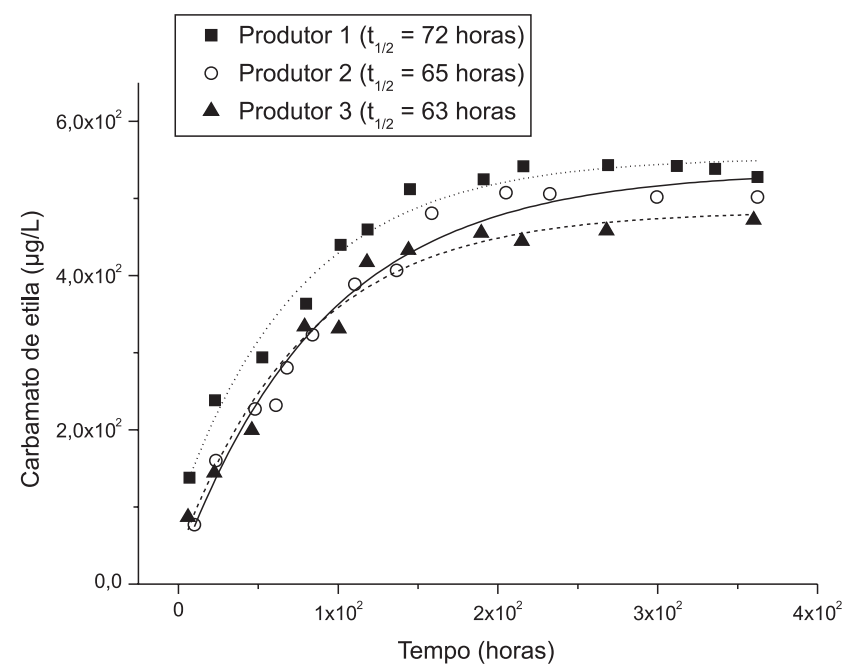

Figura 4. Carbamato de etila $(\mu g / L)$ em função do tempo $(h)$ em aguardentes oriundas de três diferentes produtores, a temperatura ambiente $\left(27 \pm 2{ }^{\circ} \mathrm{C}\right)$ : produtor 1, $\mathbf{\square}$; produtor $2, \bigcirc$ e produtor $3, \mathbf{\Delta}$, coletadas in loco

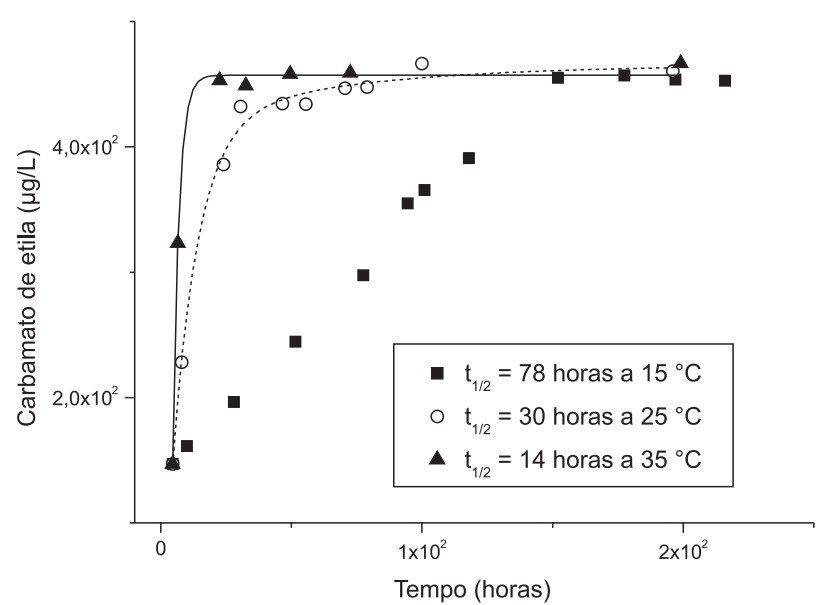

Figura 5. Carbamato de etila $(\mu \mathrm{g} / L)$ em função do tempo $(h)$ analisado nas temperaturas $15{ }^{\circ} \mathrm{C}(\mathbf{\square}), 25{ }^{\circ} \mathrm{C}(\mathrm{O})$ e $35{ }^{\circ} \mathrm{C}(\mathbf{\Delta})$ para aguardentes de um mesmo produtor, coletadas in loco

Considerando-se diferentes amostras, do mesmo produtor ou não, foi possível calcular o valor de $k_{o b s}$, expresso pela sua média, como sendo da ordem de $(3,3 \pm 0,5) \times 10^{-5} / \mathrm{s}$ a $25^{\circ} \mathrm{C}$

Os parâmetros de ativação $\Delta H^{*}, \Delta \mathrm{S}^{*}$ e $\Delta \mathrm{G}^{*}$ calculados, para uma amostra de aguardente coletada de um mesmo produtor, são respectivamente, $34 \mathrm{kcal} / \mathrm{mol}$; $-69 \mathrm{cal} / \mathrm{mol} \mathrm{K}$ e $54 \mathrm{kcal} / \mathrm{mol}$.

\section{CONCLUSÕES}

O carbamato de etila não foi completamente eliminado por um processo de redestilação em laboratório. Observou-se, entretanto, uma redução de até $92,5 \%$ da sua concentração original. A formação de CE ocorre principalmente após a destilação, $\operatorname{com} k_{o b s}=(3,3 \pm 0,5) \mathrm{x}$ $10^{-5} / \mathrm{s}$ a $25^{\circ} \mathrm{C}$, e parâmetros de ativação $\Delta H^{*} 34 \mathrm{kcal} / \mathrm{mol}$, $\Delta S^{*}-69 \mathrm{cal} / \mathrm{mol} \mathrm{K}$ e $\Delta G^{*} 54 \mathrm{kcal} / \mathrm{mol}$. Assim, os resultados experimentais, embora restritos à fração coração, confirmam que uma redestilação seria um recurso alternativo para a redução deste contaminante em etanol e em bebidas destiladas.

\section{AGRADECIMENTOS}

Ao CNPq, à CAPES e FAPESP pelo apoio financeiro. 


\section{REFERÊNCIAS}

1. Madrera, R. R.; Valles, B. S.; Food Control 2009, 20, 139.

2. Fu, M.; Liu, J.; Chen, Q.; Liu, X.; He, G.; Chen, J.; Int. J. Food Sci. Technol. 2010, 45, 1297.

3. Weber, J. V.; Sharypov, V. I.; Environ. Chem. Lett. 2009, 7, 233.

4. Zimmerli, B.; Schlatter, J.; Mutat. Res. 1991, 259, 325.

5. Lachenmeier, D. W.; Lima, M. C. P.; Nóbrega, I. C. C.; Pereira, J. A. P.; Kerr-Corrêa, F.; Kanteres, F.; Rehm, J.; BMC Cancer 2010, $10,1$.

6. Europa, European Food Safety Authority; The EFSA J. 2007, 551, 1.

7. Bruno, S. N. F.; Vaitsman, D. S.; Kunigami, C. N.; Brasil, M. G.; Food Chem. 2007, 104, 1345.

8. Nóbrega, I. C. C; Pereira, J. A. P.; Paiva, J. E.; Lachenmeier, D. W.; Food Chem. 2009, 117, 693.

9. Battaglia, R.; Conacher, H. B. S.; Page, B. D.; Food Addit. Contam. 1990, 7, 477.

10. Brasil, Ministério da Agricultura Pecuária e Abastecimento; Instrução Normativa $n^{\circ} 13$, de 29/6/2005, Diário Oficial da União, 30/06/2005, seção 1, p. 3.

11. Brasil, Ministério da Agricultura Pecuária e Abastecimento; Instrução Normativa n 21, de 19/7/2010, Diário Oficial da União, 20/07/2010, seção 1, p. 8.

12. Uthurry, C. A.; Lepe, J. A. S.; Lombardero, J.; Del Hierro, J. R.; Food Chem. 2006, 94, 262.

13. Ought, C. S.; J. Agric. Food Chem. 1976, 24, 323.

14. Daudt, C. E.; Ough, C. S.; Stevens, D.; Herraiz, T.; Am. J. Enology and Viticulture 1992, 43, 318.

15. Ough, C. S.; Crowell, E. A.; Gutlove, B. R.; Am. J. Enology and Viticulture 1988, 39, 239.

16. Mackenzie, W. M.; Clyne, A. H.; Macdonald, L. S.; J. Inst. Brewing 1990, 96, 223.

17. Stevens, D. F.; Ough, C. S.; Am. J. Enology and Viticulture 1993, 44, 309.

18. Ough, C. S.; Crowell, E. A.; Mooney, L. A.; Am. J. Enology and Viticulture 1988, 39, 243.

19. Ough, C. S.; Stevens, D. F.; Almy, J.; Am. J. Enology and Viticulture 1989, 40, 219.
20. Hasnip, S.; Caputi, A.; Crews, C.; Brereton, P.; Food Addit. Contam. 2004, 21, 1155.

21. Lachenmeier, D. W.; Schehl, B.; Kuballa, T.; Frank, W.; Senn, T.; Food Addit. Contam. 2005, 22, 397.

22. Tegmo-Larsson, I. M.; Henick-Kling, T.; Am. J. Enology and Viticulture 1990, 41, 269.

23. Cook, R.; McCaig, N.; McMillan, J. M. B.; Lumsden, W. B.; J. Inst. Brewing 1990, 96, 233 .

24. Riffkin, H. L.; Wilson, R.; Howie, D.; Muller, S. B.; J. Inst. Brewing 1989, 95, 115.

25. Jones, D. A.; Phytochemistry 1998, 47, 155.

26. Andrade-Sobrinho, L. G.; Capellini, L. T. D.; Silva, A. A.; Galinaro, C. A.; Buchviser, S. F.; Cardoso, D. R.; Franco, D. W.; Quim. Nova 2009, $32,116$.

27. Andrade-Sobrinho, L. G.; Boscolo, M.; Lima-Neto, B. S.; Franco, D. W.; Quim. Nova 2002, 25, 1074.

28. Ribani, M.; Bottoli, C. B.; Collins, C. H.; Jardim, I. C. S. F.; Melo, L. F.; Quim. Nova 2004, 27, 771.

29. MaCabe, W. L.; Smith, J. C.; Harriott, P.; Unit Operations of Chemical Engineering, $5^{\text {th }}$ ed., McGraw-Hill: New York, 1993.

30. Simoni, D. A.; Andrade, J. C.; Faigle, J. F. G.; Simoni, J. A.; Quim. Nova 2002, 25, 1034.

31. Laidler, K. J.; Chemical Kinetics, $3^{\text {rd }}$ ed., McGraw-Hill: New York, 1987.

32. Taki, N.; Iamura, L.; Takere, S.; Kobashi.; Jpn. J. Toxicol. Environ. Health 1992, 38, 498.

33. Monteiro, F. F.; Trousdale, E.; Bisson, L. F.; Am. J. Enology and Viticulture 1989, 40, 1.

34. Bizelli, L. C.; Ribeiro, C. A. F.; Novaes, F. V.; Sci. Agric. 2000, 57, 623.

35. Rota, M. B.; Faria, J. B.; Alim. Nutr. 2009, 20, 121.

36. Franco, A. C.; Rota, M. B.; Faria, J. B.; Alim. Nutr. 2009, 20, 331.

37. Park, S. R.; Ha, S. D.; Yoon, J. H.; Lee, S. Y.; Hong, K. P.; Lee, E. H.; Yeom, H. J.; Yoon, N. G.; Bae, D. H.; Food Control 2009, 20, 946.

38. Aylott, R. I.; Cochrane, G. C.; Leonard, M. J.; McDonald, L. S.; MacKenzie, W. M.; McNeish, A. S.; J. Inst. Brewing 1990, 96, 213.

39. Lawrence, J. F.; Page, B. D.; Conacher, H. B. S.; Adv. Environ. Sci. Technol. 1990, 23, 457. 